\title{
LA REFORMA DEL PRESIDENCIALISMO EN AMÉRICA LATINA. HACIA UN MODELO HÍBRIDO*
}

\author{
The Reform of Presidentialism in Latin America. Towards a Hybrid Model
}

\section{Gabriel L. Negretto ${ }^{1}$}

\begin{abstract}
Resumen: Este artículo analiza los cambios constitucionales que han alterado el poder de los presidentes en América Latina entre 1978 y 2014. Se argumenta que las tendencias de reforma en esta materia se han movido en direcciones opuestas. Se han incorporado reglas que reducen la probabilidad de que el presidente cuente con un apoyo mayoritario en el Congreso, con otras que incrementan su poder de negociar con los legisladores. Por otra parte, mientras muchas reformas se orientan a fortalecer la capacidad del Congreso para supervisar las facultades de gobierno del Ejecutivo, se adoptaron otras que concentran Poder Legislativo en manos del presidente. En este diseño híbrido, las reformas adoptadas coexisten en tensión y tienen un impacto contradictorio en el poder real de los presidentes, de modo tal que es tan incorrecto hablar de un avance del hiperpresidencialismo como de una disminución sistemática del poder de los presidentes en todas sus dimensiones.
\end{abstract}

Palabras Clave: poder presidencial, separación de poderes, reforma constitucional, América Latina.

Summary: This article analyzes the constitutional changes that have altered the power of presidents in Latin America between 1978 and 2014. It argues that reform trends in this matter have moved in opposite directions. Rules that reduce the likelihood that the president will have majority support in Congress have been incorporated, along with other rules that increase the president's power to negotiate with legislators. On the other hand, while many reforms are aimed at strengthening the capacity of the Congress to supervise the governing powers of the Executive, other reforms that concentrate Legislative Power in the hands of the president were adopted. In this hybrid design, the adopted reforms coexist in tension and have a contradictory impact on the real power of the presidents, in such a way that it is as incorrect to speak of an advance of hyperpresidentialism as of a systematic reduction of the power of the presidents in all its dimensions.

Key words: presidential power, separation of powers, constitutional reform, Latin America

\footnotetext{
* Artículo recibido el 30/11/2017 y aceptado el 22/03/2018.

1 Centro de Investigación y Docencia Económicas. División de Estudios Políticos, México. Correo electrónico: gabriel.negretto@cide.edu
} 


\section{Introducción}

Casi todas las reformas constitucionales adoptadas desde 1978 afectaron, de una manera u otra, la estructura y poderes del Ejecutivo. La evaluación de los cambios ocurridos en esta área de diseńo ha dado lugar a interpretaciones disímiles. Algunos estudios provenientes del derecho constitucional comparado indican que la estructura del poder presidencial se ha mantenido intacta o ha tendido a fortalecerse. Por otro lado, análisis empíricos provenientes de la ciencia política señalan que, en comparación con épocas pasadas, el poder de los presidentes encuentra hoy mayores límites, como resultado de la creciente fragmentación partidaria que fuerza a los ejecutivos a negociar con partidos opositores en el Congreso para legislar. Ambas interpretaciones parten de una evaluación parcial tanto de las transformaciones constitucionales que han tenido lugar en la región como de las distintas dimensiones que abarca la estructura y el ejercicio del poder presidencial.

Este artículo analiza los cambios constitucionales que han alterado el poder de los presidentes en América Latina entre 1978 y 2014. Argumentaré que las tendencias de reforma en esta materia se han movido en direcciones opuestas. Se han incorporado reglas que reducen la probabilidad de que el presidente cuente con un apoyo mayoritario en el Congreso, con otras que incrementan su poder de negociar con los legisladores. Por otra parte, mientras muchas reformas se orientan a fortalecer la capacidad del Congreso para supervisar las facultades de gobierno del Ejecutivo, se adoptaron otras que concentran poder legislativo en manos del presidente. En este diseño híbrido, las reformas adoptadas coexisten en tensión y tienen un impacto contradictorio en el poder real de los presidentes, de modo tal que es tan incorrecto hablar de un avance del hiperpresidencialismo como de una disminución sistemática del poder de los presidentes en todas sus dimensiones.

Este trabajo se divide en tres partes. En primer lugar, se discuten las interpretaciones que se han elaborado sobre la evolución reciente del poder presidencial en América Latina. En una segunda sección se analizan las reformas ocurridas en la estructura del poder presidencial en tres dimensiones: poderes partidarios y electorales, poderes de gobierno y poderes legislativos. Luego se examina cómo este diseño contradictorio ha afectado el ejercicio de poder de los presidentes en la práctica. Finalmente, concluiré con una breve reflexión acerca de la relación entre poder presidencial y desempeño de las nuevas democracias en la región.

\section{Interpretaciones del poder presidencial}

Los padres fundadores de las repúblicas hispanoamericanas adoptaron sistemas presidenciales que, si bien se inspiraron en la idea de separación de poderes, se apartaron del modelo original de frenos y contrapesos que los federalistas plasmaron en la constitución de Filadelfia. En comparación con el régimen constitucional de los Estados Unidos, los presidentes de América Latina han tenido considerablemente más atribuciones para sancionar o modificar la legislación, interferir en las funciones 
judiciales y suspender la constitución en situaciones de emergencia (véase Negretto, 2003; Cheibub, Elkins y Ginsburg, 2012). Si bien este contraste de diseños originales es real, el análisis de la evolución reciente del poder presidencial en la región se ha prestado a interpretaciones contrastantes. Mientras ciertos autores continúan utilizando la idea de "hiperpresidencialismo" para caracterizar el poder actual de los presidentes, otros indican que este ha sufrido importantes transformaciones durante la última etapa democrática.

En un estudio reciente, Gargarella (2013) afirma que las reformas constitucionales ocurridas en las últimas décadas en América Latina han aumentado consistentemente las facultades del Ejecutivo, a pesar de algunas pequeñas revisiones en la dirección opuesta. En su visión, un elemento constante del constitucionalismo latinoamericano es que, a pesar del intento frecuente de ampliar los derechos ciudadanos, la parte orgánica de la constitución o "sala de máquinas" del poder se ha mantenido intacta desde la segunda mitad del siglo XIX. Si bien Gargarella reconoce que en algunos casos se ha fortalecido el poder de los jueces o de los legisladores para supervisar al Ejecutivo, en su opinión no es correcto pensar que las reformas que parecen atenuar las atribuciones del Ejecutivo hayan tenido algún efecto significativo. En su interpretación, un análisis dinámico y contextual de la constitución mostraría que la estructura de poder presidencial ha permanecido inalterada y que, en los hechos, se sigue imponiendo sobre las demás ramas de gobierno (Gargarella, 2013, pp. 158-159).

La interpretación de Gargarella de asemeja a los argumentos que expusiera Guillermo O’Donnell a comienzos de los años 1990, cuando señalaba que, mientras la democracia electoral se expandía en América Latina, la misma no cumplía, en los hechos, con la rendición de cuentas horizontal que supone tener un sistema de separación de poderes (O’Donnell, 1994). Observaba que muchas de las nuevas democracias de la región tenían un carácter "delegativo", debido a que los presidentes, una vez electos, gobernaban sin rendir cuentas ante la legislatura o ante el Poder Judicial. Si bien O’Donnell tomaba en cuenta el creciente uso de la legislación por decreto que por entonces hacían los presidentes de Argentina, Brasil y Perú, su interpretación enfatizaba la importancia que tenían las convenciones no escritas para entender este ejercicio del poder. En la ciencia política, sin embargo, la visión de O'Donnell y, por extensión, la de Gargarella, ha sido cuestionada por una larga serie de estudios de carácter empírico y comparativo acerca de las relaciones entre poderes en América Latina.

Los primeras críticas a la visión tradicional del presidencialismo latinoamericano surgieron de estudios sobre las relaciones Ejecutivo-Legislativo. Estos señalaron que no solo la variación del poder real de los presidentes es amplia entre países y en el tiempo, sino que ni siquiera es cierto que los presidentes dotados de poderes "imperiales" gobiernen de manera unilateral. Incluso poderes que parecen muy impresionantes en el papel, como los poderes de decreto, pueden verse limitados en la práctica por la interacción que tienen los mismos con el poder partidario de los presidentes en el Congreso (ver Mainwaring y Shugart, 1997; Archer y Shugart, 1997). En particular, un presidente minoritario que decida utilizar sus poderes de decreto 
de manera confrontacional y sin formar coaliciones legislativas para compensar su debilidad fracasaría en su estrategia, como lo demuestra el caso de Collor de Melo en Brasil (ver Amorim Neto, 2002). Por otra parte, varios trabajos mostraron que tanto la creación como el uso de los poderes de decreto era tolerado e incluso promovido por los propios legisladores (ver Shugart, 1998; Carey y Shugart, 1998; Morgenstern, 2002; Morgenstern y Cox, 2002; Negretto, 2004).

Los estudios acerca del Poder Judicial se movieron en una dirección similar, argumentando que los constreñimientos que los jueces ponen al Ejecutivo varían ampliamente entre países, dependiendo tanto de sus poderes formales como de las condiciones políticas en las que se ejercen. En este sentido, por ejemplo, los modelos estratégicos de comportamiento judicial mostraron cómo los jueces constitucionales tienen mayores incentivos a limitar el poder de los ejecutivos a medida que la competencia electoral aumenta y el poder político se dispersa, como ha ocurrido en la mayor parte de las nuevas democracias de América Latina (ver Helmke, 2005, RíosFigueroa, 2007).

Hay dos problemas que se entrecruzan en estas evaluaciones. El primero es la distinción entre facultades de jure y de facto del presidente. En algunos países, como Chile o Colombia, las facultades formales de los presidentes en las áreas de gobierno, legislación y emergencias han sido históricamente muy fuertes. Sin embargo, en la práctica, no siempre demostraron ser efectivas (ver Archer y Shugart, 1997). En contraposición, en países como México o Venezuela, e incluso en los Estados Unidos, en algunas coyunturas, los presidentes han disfrutado de más influencia sobre la legislación y la creación de leyes que lo que se esperaría de las prerrogativas formales enumeradas en la Constitución (ver Weldon, 1997; Crisp, 1998). El poder real de los presidentes resulta de una interacción compleja entre sus atribuciones formales, las convenciones constitucionales no escritas, las características del sistema de partidos y de la organización partidaria y la fortaleza de otras instituciones. También importan factores extra-institucionales, como son las preferencias cambiantes de los actores políticos y las características del contexto social y económico. Si bien la mayor parte de las interpretaciones del poder presidencial tienden a enfatizar su implementación efectiva, no siempre queda explícito en el análisis los distintos componentes que determinan el poder real de los presidentes.

Por otra parte, y lo que es más importante para los fines de este trabajo, el poder de los presidentes no se puede concebir como un conjunto de prerrogativas que se puedan agregar simplemente en una única dimensión. Tanto quienes señalan la continuidad del hiperpresidencialismo como quienes indican el surgimiento de un poder más limitado y compartido, suelen enfocarse en ciertos poderes en particular, como son los poderes de legislación. Sin embargo, el poder presidencial tanto desde el punto de vista formal como real, abarca distintas atribuciones en materia partidaria, de gobierno o legislativa. Y resulta que el grado de poder que el Ejecutivo disfruta en una de estas áreas no es necesariamente consistente con su poder en otra diferente.

Para ilustrar este contraste, basta comparar los poderes presidenciales en el siglo XIX y XX. Durante el siglo XIX y hasta las primeras décadas del siglo XX, los ejecutivos de América Latina solían ser poderosos como líderes del partido gober- 
nante, tenían poderes unilaterales para conducir sus gabinetes y fuertes prerrogativas en situaciones de emergencia. Al mismo tiempo, tenían pocas facultades formales para sancionar legislación o inducir a los legisladores a aprobar los cambios que ellos deseaban hacer a las leyes vigentes (ver Negretto, 2009a; 2013a). Desde mediados a fines del siglo XX y hasta el presente, como veremos, se observa una tendencia opuesta. Los presidentes perdieron facultades partidarias, de emergencia y gobierno, mientras que ganaron facultades legislativas proactivas considerables.

\section{Reformas al poder presidencial}

El poder presidencial es un concepto multidimensional, ya que engloba el poder del Ejecutivo en sus distintos papeles como jefe de partido, jefe de Estado, jefe de gobierno y colegislador ${ }^{2}$. Las facultades electorales y partidarias se refieren al poder de los presidentes como líderes de sus partidos y de mayorías legislativas. Las facultades de gobierno aluden al poder de los presidentes para conducir a sus gabinetes y hacer nombramientos en la administración y otras instituciones. Las facultades legislativas se refieren a la capacidad institucional del Ejecutivo de influir en la creación y el cambio de leyes. Analizaré las reformas ocurridas en cada una de estas dimensiones por separado ${ }^{3}$.

\subsection{Facultades electorales y partidarias}

Los presidentes son comparativamente más poderosos si sus partidos pueden obtener una mayoría o una representación cercana a la mayoría en el Congreso, si gozan de un control centralizado sobre sus partidos y si disponen de un horizonte de largo plazo para negociar con los legisladores. Las disposiciones legales que afectan estos poderes son la fórmula para elegir el presidente, el ciclo electoral, la duración del período presidencial, la norma sobre reelección y las leyes que reglamentan la naturaleza partidaria del voto.

Los estudios institucionales comparados indican que los presidentes tienden a obtener el apoyo de una mayoría en el Congreso cuando son elegidos por mayoría relativa y las elecciones legislativas coinciden con la contienda presidencial. Por el contrario, la posibilidad de contar con mayorías legislativas disminuye cuando los presidentes son elegidos por fórmulas que requieren un umbral mínimo de votos o las elecciones presidenciales y legislativas no concurren en el tiempo y los legisladores son electos por una fórmula de representación proporcional. Esto es así pues las formulas de umbral mínimo para elegir presidente y los sistemas proporcionales para elegir legisladores tienden a pluralizar la competencia partidaria y a incrementar la fragmentación del sistema de partidos (ver Shugart y Carey, 1992; Jones, 1995, 1997; Negretto, 2007).

2 No incluiré aquí el análisis de las facultades en casos de emergencia. A pesar de ser muy importantes, estas facultades no estuvieron sujetas a tantos cambios sustantivos como las demás en las décadas recientes 3 Para un análisis más detallado de estas reformas, ver Negretto (2013). 
Las fórmulas de mayoría relativa para elegir presidentes fueron una vez predominantes en América Latina (en las décadas de 1950 y 1960), con frecuencia en combinación con elecciones legislativas concurrentes. Sin embargo, como resultado de las reformas constitucionales implementadas desde fines de la década de 1970, la mayoría de los países requieren ya sea una mayoría absoluta (más del 50\%) o una mayoría relativa calificada (un umbral mínimo de votos por debajo de 50\%, pero por encima de algún mínimo, como, por ejemplo, 20\%) para ganar una elección presidencial.

Específicamente, y como se muestra en la Tabla 1, hubo quince modificaciones en la fórmula para elegir al presidente entre 1978 y 2014 . Ocho de estas reformas reemplazaron las fórmulas de mayoría relativa por fórmulas de mayoría absoluta (exigencia de un umbral mínimo de votos mayor al 50\%) o de mayoría relativa calificada (exigencia de un umbral mínimo de votos inferior al $50 \%$, pero que oscila generalmente entre el 30 y el $45 \%$ ). En tres casos, las elecciones presidenciales directas por mayoría absoluta ya existían, pero una segunda ronda de votación entre los dos candidatos más votados reemplazó la participación del Congreso para determinar el resultado cuando ningún candidato alcanzaba el umbral requerido en primera instancia.

Tabla 1. Fórmula de umbral mínimo para elegir presidente, 1978-2014

\begin{tabular}{lll}
\hline País & Reformas & $\begin{array}{l}\text { Establece o Amplía Umbral } \\
\text { Mínimo de Votos }\end{array}$ \\
\hline ARG & 1994 & $\mathrm{Si}$ \\
\hline BOL & 2009 & $\mathrm{No}(-)$ \\
\hline BRA & 1988 & $\mathrm{Si}$ \\
\hline COL & 1991 & $\mathrm{Si}$ \\
\hline CHI & 1980 & $\mathrm{No} \mathrm{(=)}$ \\
\hline ECU & 1978 & $\mathrm{Si}$ \\
\hline ESA & 1998 & $\mathrm{No}(-)$ \\
\hline GUA & 1983 & $\mathrm{No}(=)$ \\
\hline & 1985 & $\mathrm{No}(=)$ \\
NIC & 1995 & $\mathrm{Si}$ \\
\hline PER & 2000 & $\mathrm{No}(-)$ \\
\hline DOM & 2014 & $\mathrm{No}(-)$ \\
\hline URU & 1979 & $\mathrm{Si}$ \\
\hline Total & 1994 & $\mathrm{Si}$ \\
\hline & 1996 & $\mathrm{Si}$ \\
\hline
\end{tabular}

\section{(-) Reduce umbral de votos}

(=) Mantiene umbral minimo preexistente

Fuente: Negretto (2006; 2013a). 
Sólo en cuatro casos, fórmulas menos restrictivas se reemplazaron por otras más restrictivas. En primer lugar, Ecuador adoptó, en 1998, elecciones presidenciales por mayoría relativa calificada de votos después de haber usado una fórmula de mayoría absoluta desde 1979. En segundo lugar, Nicaragua redujo, en el año 2000, el umbral de votos necesarios para ganar la elección presidencial de $45 \%$ a $40 \%$, pudiendo reducirse incluso al $35 \%$ si la diferencia con el segundo más votado fuese de al menos 5 puntos porcentuales. Bolivia cambió en 2009 de una fórmula de mayoría absoluta con una segunda vuelta de elecciones en el Congreso a una fórmula de mayoría relativa calificada con un umbral mínimo de votos del $40 \%$ y una diferencia mínima de $10 \%$ sobre el segundo candidato más votado. Por último, Nicaragua eliminó en 2014 la mayoría relativa calificada y restauró la vieja fórmula de mayoría relativa que existió en ese país hasta la reforma de 1995.

Para el año 2014, seis países en toda la región (Honduras, México, Nicaragua, Panamá, Paraguay y Venezuela) elegían a sus presidentes por la regla de mayoría relativa. De esos, solo cuatro (Honduras, Nicaragua, Panamá y Paraguay) tienen elecciones concurrentes para el Congreso. Además, y debido a una serie de reformas electorales introducidas al sistema para elegir a los diputados desde las primeras décadas del siglo XX, todas las cámaras bajas en la región (con la excepción parcial de México y Venezuela) hoy se eligen usando diferentes variantes de fórmulas de representación proporcional.

Si estas reformas producen los efectos esperados, la combinación de presidentes elegidos por fórmulas que requieren un umbral mínimo de votos y legisladores elegidos por representación proporcional, tendría que resultar en una mayor fragmentación del número de partidos que compiten y ganan bancas legislativas. Esto, a su vez, haría más probable que los presidentes carezcan de apoyo mayoritario en el Congreso y deban negociar con partidos opositores e incluso integrarlos a coaliciones formales de gobierno para poder legislar.

Este potencial debilitamiento de la capacidad de los presidentes para legislar se vería reforzado por otro grupo importante de reformas recientes que adoptan diversas formas de voto personalizado. Estas reformas han sustituido sistemas de listas cerradas de partido por listas abiertas, listas flexibles (también llamadas listas cerradas pero no bloqueadas), sistemas de voto personal en distritos plurinominales, y sistemas mixtos que combinan una votación personalizada en distritos uninominales con votación por listas cerradas de partido en distritos plurinominales (ver Negretto, 2009b). Estas reglas, en comparación con aquellas en que los líderes partidarios controlan la nominación de candidatos por medio de listas cerradas, tienden a limitar el poder disciplinario de los jefes de bancada, haciendo que los presidentes deban negociar sus agendas no solo con la oposición, sino también dentro de sus propios partidos (ver Carey y Shugart, 1995).

Los potenciales efectos de otras reformas electorales apuntan en una dirección diferente. Mientras que los períodos presidenciales podían ser de hasta 8 años en las primeras décadas del siglo XX, las reformas recientes los han reducido a 4 o 5 años. Sin embargo, también se han modificado las normas que reglamentan la reelección del presidente, haciéndolas más permisivas (véase Zovatto y Orozco Henriquez, 2008; Negretto, 2009b; 2013a). La mayoría de las reformas constitucionales 
en América Latina han relajado las normas de reelección presidencial, pasando de la proscripción absoluta o de la reelección después de un período a una sola reelección consecutiva. En contraposición con la reducción del mandato, la posibilidad de reelección consecutiva, incluso limitada a dos períodos, aumenta la probabilidad de que los presidentes en ejercicio extiendan su período en el cargo.

\section{Tabla 2. Permisividad de la reelección presidencial, 1978-2014}

\begin{tabular}{|c|c|c|}
\hline País & Reforma & $\begin{array}{l}\text { Mayor } \\
\text { Permisividad }\end{array}$ \\
\hline ARG & 1994 & $\mathrm{Si}$ \\
\hline $\mathrm{BOL}$ & 2009 & $\mathrm{Si}$ \\
\hline BRA & 1998 & $\mathrm{Si}$ \\
\hline \multirow{2}{*}{$\mathrm{COL}$} & 1991 & No \\
\hline & 2004 & $\mathrm{Si}$ \\
\hline \multirow{3}{*}{ ECU } & 1978 & No \\
\hline & 1995 & $\mathrm{Si}$ \\
\hline & 2008 & $\mathrm{Si}$ \\
\hline GUA & 1985 & No \\
\hline \multirow{3}{*}{ NIC } & 1987 & $\mathrm{Si}$ \\
\hline & 1995 & No \\
\hline & 2014 & $\mathrm{Si}$ \\
\hline PAR & 1992 & No \\
\hline \multirow{2}{*}{ PER } & 1993 & $\mathrm{Si}$ \\
\hline & 2000 & No \\
\hline \multirow{2}{*}{ REP DOM } & 1994 & No \\
\hline & 2002 & $\mathrm{Si}$ \\
\hline \multirow{2}{*}{ VEN } & 1999 & $\mathrm{Si}$ \\
\hline & 2009 & $\mathrm{Si}$ \\
\hline Total & 19 & 12 \\
\hline
\end{tabular}

Fuente: Negretto (2009b; 2011; 2013a).

Como muestra la Tabla 2, de diecinueve modificaciones que se introdujeron en las normas de reelección presidencial de 1978 a 2014, doce resultan más permisivas y siete menos permisivas. Esta tendencia no se hizo manifiesta inicialmente. De 1978 a 1993, varias de las nuevas constituciones restablecieron la tradicional regla de reelección después de un período o bien adoptaron la fórmula más severa de proscripción absoluta de reelección, como fue el caso de Guatemala en 1985 y Colombia en $1991^{4}$. Desde 1993, sin embargo, se observa una tendencia contraria, al

4 Honduras también adopta la proscripción absoluta en 1982, pero esta provenía de la Constitución de 1965. 
aumentar el número de constituciones que permiten una reelección consecutiva. Por otra parte, la autorización de reelecciones presidenciales ilimitadas, que en el pasado era típica de los regímenes autoritarios, fue adoptada en Venezuela, en 2009 y, más recientemente en Nicaragua, en 2014.

Cuando la norma de reelección presidencial permite períodos presidenciales consecutivos, es probable que se vea más limitada la rotación y alternancia en el Ejecutivo. La posibilidad de reelección consecutiva puede tener también un impacto indirecto en las facultades partidarias del presidente. En igualdad de condiciones, un presidente que puede postularse para su reelección es probable que tenga más poder de negociación con los legisladores que un presidente que no puede ser reelecto.

Este análisis apunta, entonces, a tendencias inconsistentes de reforma en la dimensión electoral del poder presidencial. Por un lado, la adopción de reglas electorales más incluyentes y competitivas reduciría la probabilidad de que los presidentes ganen una mayoría en el Congreso y cuenten con el apoyo de partidos cohesivos. Por el otro lado, sin embargo, la duración del plazo que están los presidentes en funciones puede aumentar, debido a normas más permisivas de reelección presidencial. Esto limitaría la rotación de los individuos y los partidos en el Poder Ejecutivo y fortalecería la posición de los presidentes en las negociaciones legislativas.

\subsection{Facultades de gobierno}

Tradicionalmente, los presidentes en América Latina han disfrutado un alto grado de independencia del Congreso en materia de formación, coordinación y cambio de los gabinetes. Desde la década de 1850, ninguna constitución en la región ha exigido la intervención del Congreso o de una de sus cámaras para confirmar el nombramiento del gabinete de ministros. Un procedimiento denominado interpelación parlamentaria ha formado parte de la mayoría de las constituciones en América Latina desde comienzos del siglo XIX. Este procedimiento, sin embargo, no implica normalmente la posibilidad de forzar la renuncia de los ministros; sólo confiere a los legisladores la autoridad de convocar a los ministros del gabinete a una sesión del Congreso para brindar información sobre un área en particular bajo su responsabilidad.

Sin embargo, con el correr del tiempo, varias constituciones en América Latina impusieron mayores restricciones sobre las facultades de gobierno de los presidentes, permitiendo a las legislaturas no sólo interrogar a los ministros del gabinete, sino también censurarlos, a veces con efectos vinculantes 5 . Esta tendencia ha crecido desde 1978. La incorporación de mecanismos de censura se relaciona indirectamente con los debates que tuvieron lugar durante finales de la década de 1980 y comienzos de la década de 1990 en países como Brasil, Argentina y Bolivia acerca de los méritos de pasar de un régimen presidencial a uno mixto con un presidente elegido independientemente y un jefe de gobierno responsable ante la asamblea legislativa (ver Nino, 1992). Ningún país sancionó tal reforma, pero varias modificaciones constitucionales en la región fortalecieron los controles del Congreso sobre los gabinetes, con frecuencia con la intención de introducir características parlamentarias dentro de la estructura de un régimen presidencial.

5 Para una evaluación histórica de estas reformas, ver Stokes (1945). 
Tabla 3. Control del Congreso sobre el gabinete presidencial, 1978-2014

\begin{tabular}{lll}
\hline País & Reforma & $\begin{array}{l}\text { Establece o } \\
\text { Incrementa } \\
\text { Control }\end{array}$ \\
\hline ARG & 1994 & $\mathrm{Si}$ \\
\hline $\mathrm{COL}$ & 1991 & $\mathrm{Si}$ \\
\hline $\mathrm{CHI}$ & 2005 & $\mathrm{Si}$ \\
\hline ECU & 1978 & $\mathrm{Si}$ \\
\hline ESA & 1998 & $\mathrm{No} \mathrm{(-)}$ \\
\hline NIC & 1983 & $\mathrm{Si}$ \\
\hline PAR & 1995 & $\mathrm{Si}$ \\
\hline PER & 1992 & $\mathrm{Si}$ \\
\hline VEN & 1979 & $\mathrm{No} \mathrm{(-)}$ \\
\hline Total & 1999 & No (-) \\
\hline
\end{tabular}

\section{(-) Disminuye control preexistente}

(=) Mantiene control preexistente

Fuente: Negretto $(2011 ; 2013 a)$.

Como muestra la Tabla 3, de un total de diez cambios sustantivos en esta área entre 1978 y 2014, el poder formal del Congreso sobre los gabinetes ha aumentado en siete 6 . Solo en tres casos (Ecuador en 1998, Perú en 1979 y Venezuela en 1999) el poder del Congreso sobre los gabinetes decreció. Como consecuencia de estas reformas y las constituciones que mantuvieron mecanismos cuasi-parlamentarios creados en el pasado (como son los casos típicos de Perú y Uruguay), en 2014, trece países en América Latina disponían de alguna forma de control político del Congreso sobre los gabinetes.

La misma tendencia se puede observar en otras áreas de las facultades de gobierno de los presidentes. Los presidentes en América Latina han tenido tradicionalmente la facultad de nombrar o al menos influir sobre el nombramiento de las autoridades locales, los jueces de las cortes constitucionales, el procurador general de la nación y los miembros de las instituciones de control. Los cambios más importantes en estas facultades se han introducido desde 1978, ya sea fortaleciendo los controles del Congreso sobre los nombramientos del Ejecutivo o eliminando completamente la influencia del presidente.

6 He considerado que es un aumento en las facultades del Congreso (y, por lo tanto, una reducción de las facultades presidenciales) adoptar un mecanismo de censura cuando este no existe, hacer menos demandantes los requisitos para iniciar una moción de censura y hacer la censura vinculante, cuando antes no lo era. He contado el mecanismo tradicional de interpelación solo cuando no existía antes de la reforma, lo que solo ocurría en Chile antes de la enmienda de 2005. 
Las medidas de descentralización política introducidas en los estados unitarios han privado a los presidentes de una fuente importante de poder y patronazgo (Grindle, 2000; Montero y Samuels, 2004; O’Neill, 2005). Tal fue el caso con la introducción de la elección popular de todos los alcaldes en Bolivia en 1994 y la elección popular de gobernadores en Venezuela en 1989, Colombia en 1991 y Paraguay en 1992. Las reformas de descentralización política también han reducido los poderes de nombramiento de los presidentes en los estados federales, donde el alcalde de la ciudad capital del país era nombrado por el presidente, como en Argentina hasta la reforma de 1994 y en México hasta la reforma de 1996.

Los poderes de nombramiento de los presidentes también se vieron reducidos como resultado de las reformas tendientes a fortalecer la independencia judicial (Rios-Figueroa, 2011). Desde las reformas de 1994 en Argentina y México, por ejemplo, los presidentes han necesitado el apoyo de una mayoría calificada del Senado (en lugar de una mayoría simple, como se exigía en el pasado) para nombrar a los jueces de la Corte Suprema. Reformas similares ocurrieron en varios países, reduciendo las facultades del presidente para nombrar al procurador general, al fiscal general y a los jefes de las instituciones de control de la administración, tales como el auditor o contralor general.

Ninguna de las reformas mencionadas en materia de gobierno y administración han alterado la estructura esencial del régimen presidencial, que se caracteriza por la duración del mandato del jefe de gobierno por un período fijo e independiente del apoyo que tenga su partido o coalición en la legislatura (ver Cheibub, 2007). Sin embargo, y comparadas con un pasado en donde la autonomía del Ejecutivo en materia de gobierno era casi absoluta, es claro que la adopción de mecanismos parlamentarios de control y la reducción de las facultades de nombramiento de los presidentes tiene el potencial de generar una distribución de facultades más equilibrada entre el Ejecutivo y las otras ramas del poder.

\subsection{Facultades legislativas}

En un fuerte contraste con lo recién explicado respecto a las facultades de gobierno, los reemplazos y las enmiendas constitucionales adoptadas desde fines de la década de 1970 han aumentado de forma significativa las facultades de los presidentes para influir en la legislación, en particular, sus capacidades para promover modificaciones en la legislación vigente. De hecho, esta tendencia comenzó mucho tiempo antes.

De la misma manera que en el modelo estadounidense, la constitución latinoamericana típica durante el siglo XIX confería al presidente una fuerte facultad reactiva (por lo general, un veto sujeto a superación por parte de una mayoría calificada en cada cámara de un Congreso bicameral), pero privaba al Ejecutivo de cualquier facultad específica para cambiar el status quo legislativo. Este modelo prevaleció en la amplia mayoría de las constituciones de América Latina hasta las primeras décadas del siglo XX. Sin embargo, desde entonces, una tendencia persistente de reformas ha fortalecido los poderes de los presidentes para promover cambios en la legislación. 
A pesar de que algunas reformas han alterado las facultades de veto de los presidentes, los cambios más importantes y frecuentes introducidos en la asignación de facultades legislativas han tenido lugar en el área del poder de agenda. La capacidad de controlar la agenda legislativa permite a los presidentes limitar el conjunto de alternativas sobre las cuales la asamblea puede elegir, el tiempo en el cual debe decidir, o ambas cosas (Carey y Shugart, 1998; Negretto, 2004). Durante todo el siglo XX, los poderes legislativos proactivos de los presidentes han aumentado constantemente en cinco áreas. Los presidentes han adquirido: (i) potestad exclusiva para presentar proyectos de ley sobre temas económicos y financieros importantes y la facultad de (ii) fijar el presupuesto; (iii) presentar proyectos de ley que se deben votar en el Congreso dentro de un plazo limitado (usualmente denominados leyes de urgencia); (iv) emitir decretos de contenido legislativo (habitualmente bajo circunstancias de urgencia extrema que hacen imposible seguir los procedimientos ordinarios de creación de leyes) y; (v) presentar propuestas de leyes a referendo popular.

Excepto por Colombia, cuya constitución de 1886 autorizaba al presidente a emitir decretos con fuerza inmediata de ley en casos de conmoción interna, ningún presidente en América Latina tenía ninguna de las facultades mencionadas para controlar la agenda legislativa a comienzos del siglo XX. Para el año 1930, las constituciones de Chile y Uruguay (dos países pioneros en fortalecer los poderes legislativos proactivos del Ejecutivo) habían dado a los presidentes facultades tales como presentar proyectos de ley de urgencia, iniciativa exclusiva sobre proyectos de leyes financieras o mayor poder sobre la elaboración y aprobación del presupuesto. El número de constituciones que confieren a los presidentes alguna forma de poder para fijar la agenda aumentó a siete para 1940, a diez en 1960 y a trece en 1980. 
Tabla 4. Reformas a los poderes legislativos del presidente, 1978-2014

\begin{tabular}{|c|c|c|}
\hline País & Reforma & $\begin{array}{l}\text { Incremento de } \\
\text { Poderes }\end{array}$ \\
\hline ARG & 1994 & $\mathrm{Si}$ \\
\hline $\mathrm{BOL}$ & 2009 & $\mathrm{Si}$ \\
\hline \multirow{2}{*}{ BRA } & * $\quad 1988$ & No \\
\hline & 2001 & No \\
\hline $\mathrm{COL}$ & 1991 & No \\
\hline $\mathrm{CHI}$ & 1980 & $\mathrm{Si}$ \\
\hline \multirow{3}{*}{ ECU } & 1978 & $\mathrm{Si}$ \\
\hline & 1983 & $\mathrm{Si}$ \\
\hline & 1998 & $\mathrm{Si}$ \\
\hline ESA & 1983 & $\mathrm{Si}$ \\
\hline $\mathrm{HON}$ & 1982 & $\mathrm{Si}$ \\
\hline MEX & 2012 & $\mathrm{Si}$ \\
\hline \multirow{2}{*}{ NIC } & $1987 *$ & No \\
\hline & 1995 & No \\
\hline PAN & 1983 & No \\
\hline PAR & $1992 *$ & No \\
\hline \multirow{2}{*}{ PER } & 1979 & $\mathrm{Si}$ \\
\hline & 1993 & $\mathrm{Si}$ \\
\hline URU & 1996 & $\mathrm{Si}$ \\
\hline VEN & 1999 & $\mathrm{Si}$ \\
\hline Total & 20 & 13 \\
\hline
\end{tabular}

${ }^{*}$ Constitución autoritaria anterior al cambio.

Fuente: Negretto (2009a; 2011; 2013a).

A pesar de que muchas constituciones autoritarias contribuyeron en el pasado a fortalecer las facultades legislativas del Ejecutivo durante períodos de dictadura civil o militar, el proceso más reciente de democratización en la región no ha revertido este legado. La mayoría de los países que reemplazaron o revisaron sus constituciones entre 1978 y 2014 han retenido fuertes facultades legislativas en manos del presidente o aumentado esas facultades. Como indica la Tabla 4, de las veinte reformas que alteraron la distribución de las facultades legislativas entre los presidentes y las asambleas, trece fortalecieron las facultades del Ejecutivo y solo siete las debilitaron en comparación con el status quo.

Vale la pena notar dos puntos acerca de esta tendencia. Primero, la mayoría de las reformas que redujeron las facultades legislativas previas de los presidentes - Brasil en 1988 y 2001, Colombia en 1991, Nicaragua en 1987 y Paraguay en 1992 - dejaron 
a los presidentes con atribuciones en materia legislativa que son todavía muy fuertes en términos comparativos. En segundo lugar, el aumento relativo en las facultades legislativas del presidente se debió exclusivamente al fortalecimiento del poder de veto del Ejecutivo en solo dos casos: El Salvador en 1983 y Uruguay en 1996. Todos los demás casos involucraron el fortalecimiento de al menos algunas de las facultades legislativas proactivas del presidente. Como resultado de estas reformas, para 2014 solo tres países en América Latina - Costa Rica, la República Dominicana y Nicaragua - tenían todavía constituciones que no otorgaban a los presidentes facultades significativas para inducir cambios legislativos.

Como veremos, la influencia real de los presidentes en la creación de leyes no puede derivar sólo de las facultades legislativas formales. Sin embargo, y desde el punto de vista de las tendencias de reforma, es innegable el contraste entre los cambios introducidos en materia de poderes de gobierno y en materia de poderes legislativos. En tanto que los primeros tienden a buscar un nuevo equilibrio de poderes, eliminando o limitando ciertos poderes tradicionales del ejecutivo, los segundos dan continuidad y refuerzan la centralidad del presidente en los regímenes constitucionales latinoamericanos.

\section{El nuevo poder presidencial en la práctica}

Desde mediados del siglo XIX hasta comienzos del siglo XX, evaluar el poder de los presidentes latinoamericanos era relativamente sencillo. Desde el punto de vista del diseño, los presidentes latinoamericanos se elegían conforme a normas electorales restrictivas, disfrutaban de fuertes facultades unilaterales de gobierno y de fuertes, pero meramente reactivas, facultades legislativas (por ejemplo, el veto). También se les confería prerrogativas excepcionales en casos de emergencia (ver Aguilar Rivera y Negretto, 2000; Negretto, 2003). De hecho, y a pesar de otras características particulares, las facultades de emergencia del Ejecutivo constituyeron durante este período la principal diferencia que uno puede observar entre las facultades formales de los presidentes en América Latina y los Estados Unidos (pero véase Cheibub, Elkins y Ginsburg, 2012).

Desde el punto de vista de la implementación, la combinación de normas electorales restrictivas, tales como las elecciones presidenciales indirectas, con la práctica informal de transferencia del poder a través de acuerdos entre elites y el control del gobierno sobre las elecciones, otorgó a los presidentes un control unificado sobre la legislatura. Cuando las fuerzas de la oposición no se sometían a los resultados electorales previamente acordados, el presidente siempre podía recurrir a sus facultades de emergencia para aplacar el disenso. Dada su supervisión de las elecciones en el Congreso y la debilidad de la oposición política, los presidentes de esta época solían ejercer influencia sobre la mayoría de las instituciones del estado, incluido el Poder Judicial.

Las reformas constitucionales adoptadas desde los comienzos del siglo XX y profundizadas después de 1978 han alterado radicalmente este escenario. Desde el punto de vista del diseño formal, las nuevas instituciones mueven al poder presi- 
dencial en direcciones potencialmente opuestas. El proceso de democratización y el surgimiento de nuevos partidos conllevaron la adopción de normas electorales más incluyentes, inicialmente fórmulas proporcionales para elegir a los diputados y, con posterioridad, desde fines de la década de 1970, fórmulas que requieren un umbral mínimo para elegir presidentes. Como resultado de las experiencias autoritarias de las décadas de los años 1950 y 1960, los reformadores en varios países buscaron reducir el poder tradicional de los presidentes proponiendo pasar de un sistema presidencial a otro semipresidencial. Ningún país abandonó finalmente la estructura presidencial de gobierno heredada del siglo XIX, pero varias reformas introdujeron instituciones parlamentarias que aumentaron los controles del Parlamento sobre el gobierno. Otras reformas limitaron sus facultades de nombramiento y fortalecieron la independencia y las facultades del Poder Judicial y las instituciones de control.

Esta tendencia no se ha mantenido, sin embargo, para todas las dimensiones del poder presidencial. Tanto antes como desde 1978, las facultades legislativas de los presidentes de América Latina han aumentado, en particular la facultad de iniciar y promover cambios en la legislación. Esta característica con frecuencia produce concentración del poder en manos del presidente, quien obtiene una ventaja importante para establecer la agenda legislativa. Al mismo tiempo, un número creciente de constituciones y enmiendas adoptadas desde comienzos de la década de 1990 han hecho que las normas de reelección presidencial sean más permisivas, autorizando por lo general a los presidentes a presentarse para una reelección consecutiva por una sola vez.

Aunque estas reformas no siempre cumplieron con los efectos esperados, su impacto sobre el poder real de los presidentes también ha sido ambivalente. Esta ambivalencia se hace manifiesta cuando observamos que es posible llegar a conclusiones diametralmente opuestas fundados en un análisis del poder fáctico de los presidentes. Como ya he señalado, Gargarella argumenta (aunque sin demostrar en un análisis empírico sistemático), que el poder de los presidentes sigue imponiéndose, en los hechos, al Poder Judicial, al Legislativo y a los organismos de control. Por otra parte, los estudios provenientes de la ciencia política muestran que el poder efectivo de los presidentes en la etapa democrática ha sido menos unilateral que en el pasado. Ambas evaluaciones son parcialmente correctas o falsas, dependiendo del caso particular y, sobre todo, de la dimensión de poder presidencial que estemos hablando.

En materia electoral, la mayor parte de las reformas ha tenido en los hechos los efectos esperados. Como resultado de la combinación de presidentes elegidos por fórmulas que requieren un umbral mínimo de votos y legisladores elegidos por representación proporcional, la fragmentación del sistema de partidos ha aumentado con el tiempo en América Latina. En particular, el número efectivo de partidos en el Congreso en toda la región pasó de un promedio de 2,7 durante el período 19401977, a 3,2 entre 1978 y 1993, y a 3,7 entre 1994 y 2009. A su vez, como consecuencia del mayor número efectivo de partidos legislativos, las presidencias minoritarias y los gobiernos divididos han sido la norma en la región desde 1978 al presente (ver Negretto, 2006). Esto implica una disminución neta del poder partidario de los presidentes, quienes se ven hoy forzados a formar coaliciones o negociar más ampliamente sus agendas legislativas con partidos de oposición para poder implementarlas. 
En cuanto a la influencia de la estructura de las listas de partido, hay un largo debate en la literatura acerca de si las mismas tienen un impacto directo sobre la disciplina y la cohesión interna de los partidos y por tanto en la capacidad que tienen los líderes de coordinar a sus legisladores en el Congreso. En un estudio reciente, Carey (2009) muestra de manera empírica e incluyendo casos latinoamericanos que, en comparación con las listas cerradas, todos los sistemas de voto personalizado disminuyen en los hechos la unidad partidaria e incrementan la competencia dentro de los partidos. Esto indica que, como consecuencia de las reformas tendientes a personalizar el voto y limitar el poder disciplinario de los líderes de partido, muchos presidentes deben hoy negociar sus agendas también al interior de sus propios partidos.

Por otra parte, también han producido el efecto esperado otras reformas electorales que, contrariamente a las mencionadas, tienden a incrementar el poder del presidente, como es el caso de la reelección presidencial consecutiva. Uno podría argumentar (como hacen con frecuencia los partidarios de la reelección presidencial consecutiva) que la mera posibilidad de ser reelegido no significa que el presidente de turno sea realmente reelecto. Sin embargo, los presidentes en ejercicio (en América Latina y en todo el mundo) gozan de una ventaja fáctica sobre sus opositores cuando pueden ser reelectos 7 . De acuerdo con David Mayhew (2008), en los Estados Unidos, los presidentes en funciones ganaron el $68 \%$ de las elecciones presidenciales en las que compitieron entre 1788 y 2004. Un fenómeno similar se puede observar en América Latina, donde los presidentes en funciones ganaron el $79 \%$ de las elecciones en las que compitieron entre 1978 y 2008. Por esta razón, si la constitución permite períodos presidenciales consecutivos, como es cada vez más común en América Latina, es muy probable que el presidente en ejercicio y su partido obtengan una ventaja electoral y se vea en los hechos limitada la rotación y la alternancia en el Ejecutivo. Este efecto, a su vez, fortalece al presidente tanto respecto de los legisladores de su propio partido como de la oposición.

Si dirigimos nuestra mirada a las relaciones entre poderes, podemos observar el efecto diferenciado de ciertas reformas dentro de un esquema cuyo conjunto sigue siendo híbrido. Por ejemplo, el impacto de las reformas recientes que limitan las facultades presidenciales sobre la conducción de los gabinetes ha sido en general modesto en sus consecuencias. Sea por restricciones formales en su implementación (como umbrales altos de votación en contextos partidarios fragmentados) o debido a los recursos políticos y económicos que los presidentes tienen a su disposición para comprar lealtades o disuadir a la oposición, los mecanismos cuasi parlamentarios no han cambiado drásticamente las facultades de gobierno de los presidentes en países que los han adoptado recientemente, como Argentina, Colombia, El Salvador, Nicaragua o Paraguay ${ }^{8}$. Por otra parte, salvo en países como Colombia, el nivel de estabilidad real de los jueces constitucionales en América Latina y su protección frente a una injerencia indebida del ejecutivo, no se corresponde con las nuevas garantías de estabilidad e

\footnotetext{
7 Las razones más importantes para esta ventaja se relacionan con los mayores recursos y aptitudes adquiridas en el cargo de quienes controlan la presidencia en el presente y la aversión al riesgo de los votantes.

8 Esto puede verificarse por el escaso número de veces que las facultades de control del Congreso se han hecho efectivas en estos países. Ver, por ejemplo, el análisis de Sarabia Better (2003, p. 73) para Colombia.
} 
independencia judicial que ofrece la constitución (ver Helmke y Rios-Figueroa, 2011). En contraste, sin embargo, las reformas que limitan las facultades del presidente de nombrar gobernadores, regidores o alcaldes, han en general reducido las capacidades de control e influencia territorial del ejecutivo. El impacto más evidente es quizás en aquellos países como Bolivia, Colombia, Paraguay o Perú, donde se estableció la elección directa de autoridades regionales que antes nombraba el presidente.

Las reformas en materia de poderes legislativos del presidente son las más complejas de evaluar en los hechos, pues el impacto real del ejecutivo como legislador es normalmente condicional al apoyo partidario que este tenga en la legislatura. Incluso en los casos más extremos, como la capacidad de emitir decretos de contenido legislativo en situaciones de emergencia, los presidentes necesitan generalmente el apoyo del Congreso para convertir esos decretos en leyes permanentes. Como expliqué anteriormente, en la América Latina contemporánea, los presidentes no disfrutan usualmente de un apoyo partidario fuerte en el Congreso. Los presidentes, sin embargo, tienen la capacidad de compensar la desventaja de su posición minoritaria forjando coaliciones legislativas formales o informales y/o usando recursos materiales o administrativos para comprar el apoyo de legisladores. Esto, entre otros factores, explica por qué hay variaciones significativas en la influencia legislativa de los presidentes tanto dentro como entre los países (véase Saiegh, 2011). No obstante, y dado que el peso del partido del presidente en el Congreso ha disminuido generalmente en toda la región, la mayoría de los estudios comparativos tienden a coincidir en que la injerencia del presidente sobre el proceso legislativo es mayor de la que tendría en ausencia de muchos de los nuevos poderes que han adquirido por medio de sucesivas reformas constitucionales (ver Morgenstern y Nacif, 2002; Shugart y Haggard, 2001).

El carácter ambivalente del poder presidencial se confirma cuando consideramos casos particulares. A pesar de que cada país y Presidencia necesita de una evaluación específica, en la mayoría de los casos el poder real de los presidentes ha variado en diferentes dimensiones, incluso dentro de un mismo país. Por ejemplo, la Constitución colombiana de 1991 redujo drásticamente las tradicionales facultades de emergencia del presidente y creó una Corte Constitucional que ha hecho valer de forma efectiva las nuevas disposiciones (Uprimny, 2004). Por otra parte, y a pesar de sus aún fuertes facultades legislativas, la capacidad del presidente de promover cambios en la legislación se ha limitado significativamente en la práctica debido a la fragmentación creciente del sistema de partidos desde 1991. No obstante, al mismo tiempo, una reforma de 2004 que permite al presidente presentarse para una reelección consecutiva ha aumentado la influencia del Ejecutivo sobre las elecciones del Congreso y ha fortalecido su poder de negociación frente a los legisladores (véase Cárdenas, Junguito y Pachón, 2006).

Después de la modificación constitucional de 1994 en Argentina, el presidente disfruta del poder formal de sancionar decretos de contenido legislativo en situaciones de necesidad y urgencia. Esta facultad fue usada de manera frecuente para controlar la agenda legislativa durante las presidencias de Menem (1995-1999), de la Rúa (1999-2001) y Néstor Kirchner (2003-2007). Al mismo tiempo, sin embargo, la misma reforma privó al presidente de la facultad de nombrar al jefe de gobierno 
de la ciudad capital y de decidir por decreto la intervención de las autoridades provinciales. Como resultado de estas reformas, desde 1996 el jefe de gobierno de la ciudad de Buenos Aires ha sido electo popularmente y hasta 2015 ningún candidato del partido del presidente ganó ese puesto (ver Negretto, 2013b). Por otra parte, y en contraste con la larga historia de abuso presidencial de esta facultad en ese país, desde la reforma de 1994 se han decretado sólo dos intervenciones federales, ambas con aprobación del Congreso?.

Es posible reproducir un análisis similar en la gran mayoría de los países de la región. Quizás los únicos países donde casi todos los analistas coinciden en observar un claro aumento del poder presidencial, tanto de jure como de facto, son aquellos (como Venezuela después de 2006 o Ecuador después de 2007) en los cuales el partido gobernante pasó a ser dominante y ha logrado controlar todas o la mayor parte de las instituciones del estado. Sin embargo, estos países no son considerados actualmente como democracias sino como autoritarismos competitivos o semi-democracias.

\section{Conclusión}

Este trabajo ha mostrado que el panorama del poder presidencial en las nuevas constituciones latinoamericanas es el de una estructura híbrida con varios componentes en tensión. Algunas partes de este diseño híbrido tienden a limitar el poder tradicional de los presidentes, mientras que otras se orientan a aumentarlo. Esta misma tensión se observa en el análisis del poder real de los presidentes cuando lo observamos en sus distintas dimensiones. Por esta razón, es posible concluir que el poder presidencial en América Latina se ha transformado desde 1978 y que ni el concepto de hiperpresidencialismo ni el de un poder presidencial atenuado es capaz de capturar esta transformación.

Es interesante observar que esta evaluación contradictoria del nuevo poder presidencial en América Latina tiene su paralelo en las caracterizaciones contrapuestas que se ofrecen hoy en día acerca de la democracia en la región. Por un lado, se ha afirmado con razón que a partir del último ciclo de expansión de la democracia, ésta ha tenido una mayor duración y estabilidad que en cualquier otra época anterior. Por otro lado, se observa que las nuevas democracias adolecen de fallas profundas en cuanto al respeto a la Constitución, la separación de poderes y la vigencia efectiva de derechos y garantías ciudadanas. Más aún, nunca como hoy se había puesto en evidencia la insuficiencia de un concepto de democracia que pone énfasis en el acceso al poder por medio de elecciones competitivas sin tomar en cuenta como se ejerce el poder luego de la elección. Dada la importancia histórica que ha tenido el poder presidencial tanto para expandir como para limitar la democracia en América Latina, no es de extrañar que el carácter bifronte del Poder Ejecutivo sea otra forma de ver los aspectos paradojales del proceso de democratización en la región.

9 Sólo durante la primer presidencia de Menem (1989-1995) se declararon 7 intervenciones, de las cuales 6 fueron por decreto del Ejecutivo. Ver Serrafero (2005). 


\section{Bibliografía}

Amorim Neto, O. (2002). "Presidential Cabinets, Electoral Cycles, and Coalition Discipline in Brazil”, en Scott Morgenstern and Benito Nacif (eds.), Legislative Politics in Latin America. Nueva York: Cambridge University Press.

Archer, R. y Shugart, M. (1997). "The Unrealized Potential of Presidential Dominance in Colombia”, en S. Mainwaring y M. Shugart (Eds.), Presidentialism and Democracy in Latin America, pp. 110-159. New York: Cambridge University Press.

Carey, John (2009). Legislative Voting and Accountability. New York: Cambridge University Press.

Carey, John y Matthew Shugart (Eds.) (1998). Executive Decree Authority. New York: Cambridge University Press.

Cárdenas, Mauricio, Roberto Junguito, y Mónica Pachón (2006). Political Institutions and Policy Outcomes in Colombia: The Effects of the 1991 Constitution. Research Network Working Paper \# R-508. Washington, DC: Inter-American Development Bank.

Cox, G. y Morgenstern, S. (2002). "Epilogue: Latin America's Reactive Assemblies and Proactive Presidents”, en S. Morgenstern y B. Nacif (Eds.) Legislative Politics in Latin America, pp. 446-468. Cambridge: Cambridge University Press.

Cheibub, José Antonio. (2007). Presidentialism, Parliamentarism, and Democracy. NuevaYork: Cambridge University Press.

Cheibub, J. A., Elkins, Z. y Ginsburg, T. (2012). "Still the Land of Presidentialism? Executives and the Latin American Constitution”, en D. Nolte y A. Schilling-Vacaflor (Eds.), New Constitutionalism in Latin America: Promises and Practices. Farham: Ashgate.

Crisp, B. (1998). "Presidential Decree Authority in Venezuela”, en C. John y M. Shugart (Eds.), Executive Decree Authority. NuevaYork: Cambridge University Press.

Gargarella, Roberto (2013). Latin American Constitutionalism, 1810-2010. NuevaYork: Oxford University Press.

Grindle, Merilee (2000). Audacious Reforms: Institutional Invention and Democracy in Latin America. Baltimore, MD: The Johns Hopkins University Press.

Helmke, G. (2005). “Enduring Uncertainty: Court-Executive Relations in Argentina During the 1990s and Beyond", en S. Levitsky y M. V. Murillo (Eds.), The Politics of Institutional Weakness. Argentine Democracy, 139-162. University Park, PA: The Pennsylvania State University Press.

Helmke, G. y Ríos-Figueroa, J. (2011). “Introduction: Courts in Latin America”, en G. Helmke y J. RiosFigueroa (Eds.), Courts in Latin America, pp. 1-26. NuevaYork: Cambridge University Press.

Mainwaring, Scott y Matthew Shugart (Eds.) (1997). Presidentialism and Democracy in Latin America. Cambridge: Cambridge University Press.

Mayhew, D. (2008). “Incumbency Advantage in US Presidential Elections: The Historical Record”, Political Science Quarterly, 123 (2), pp. 201-228.

Montero, Alfred P. y David J. Samuels (Eds.) (2004). Decentralization and Democracy in Latin America. Notre Dame: University of Notre Dame Press.

Morgenstern, S. (2002). “Towards a Model of Latin American Legislatures”, en S. Morgenstern y B. Nacif, (Eds.), Legislative Politics in Latin America, pp. 446-468. Cambridge: Cambridge University Press.

Negretto, G. y Aguilar-Rivera, J. A. (2000). "Rethinking the Legacy of the Liberal State in Latin America: The Cases of Argentina (18531-912) and Mexico (18571-910)", Journal of Latin American 
Studies 32, pp. 361-397.

Negretto, Gabriel (2013a). Making Constitutions: Presidents, Parties, and Institutional Choice in Latin America. NuevaYork: Cambridge University Press.

. (2013) "El Papel de la Constitución en la Nueva Democracia Argentina", Revista de la Sociedad Argentina de Análisis Político, 7 (2), Diciembre.

. (2011). "Shifting Constitutional Designs in Latin America: A Two-Level Explanation", Texas Law Review, 89 (7), pp. 1777-1806.

(2009a). "Political Parties and Institutional Design: Explaining Constitutional Choice in Latin America", British Journal of Political Science. Vol. 39, pp. 117-139.

(2009b). "La Reforma Electoral en América Latina. Entre el Interés Partidario y las

Demandas Ciudadanas", en A. Fontaine, C. Larroulet, J. Navarrete e I. Walter (Eds.), Reforma del Sistema Electora Chileno, pp. 63-103. Santiago de Chile: PNUD.

. (2007). "Propuesta para una reforma electoral en México", Política y Gobierno XIV(1), pp. 200-215.

. (2006). "Choosing How to Choose Presidents: Parties, Military Rulers, and Presidential Elections in Latin America”, The Journal of Politics, 68 (2) Mayo, pp. 421-433.

. (2004). "Government Capacities and Policy Making by Decree in Latin America: The

Cases of Brazil and Argentina”, Comparative Political Studies, Vol. 37, pp. 531-62.

. (2003). "Diseño Constitucional y Separación de Poderes en América Latina”, Revista Mexicana de Sociología, 65 (1), pp. 41-76.

Nino, C. (1992). "Ideas and Attempts at Reforming the Presidentialist System of Government in Latin America”, en A. Lijphart (Ed.), Parliamentary Versus Presidential Government, pp. 128-132. NuevaYork: Oxford University Press.

O’Donnell, G. (1994). "Delegative Democracy”, Journal of Democracy 5 (1), pp. 55-69.

O’Neill, Kathleen (2005). Decentralizing the State: Elections, Parties, and Local Power in the Andes. NuevaYork: Cambridge University Press.

Rios-Figueroa, J. (2007). "Fragmentation of Power and the Emergence of an Effective Judiciary in Mexico, 1994-2002”, Latin American Politics and Society, Vol. 49 (1), pp. 31-57.

. (2011). "Institutions for Constitutional Justice in Latin America", en G. Helmke y J.

Rios-Figueroa (Eds.), Courts in Latin America, pp. 27-54. NuevaYork: Cambridge University Press.

Saiegh, S. (2015). "Executive-Legislative Relations”, en Jennifer Ghandi y Ruben Ruiz-Rufino (Eds.), Routledge Handbook of Comparative Political Institutions. NuevaYork: Routledge.

Sarabia Better, Arturo (2003). Reformas políticas en Colombia: del plebiscito de 1957 al referendo de 2003. Bogotá: Grupo Editorial Norma.

Serrafero, Mario (2005). Exceptocracia: ¿Confín de la Democracia? Intervención Federal, Estado de Sitio y Decretos de Necesidad y Urgencia. Buenos Aires: Editorial Lumiere.

Shugart, M. (1995). "The Electoral Cycle and Institutional Sources of Divided Government", American Political Science Review 89(2), pp. 327-343.

. (1998). "The Inverse Relationship Between Party Strength and Executive Strength: A Theory of Politicians' Constitutional Choices", British Journal of Political Science 28(1), pp. 1-29.

Shugart, M. y Carey, J. (1992). President and Assemblies: Constitutional Design and Electoral Dynamics. Cambridge, MA: Cambridge University Press.

Shugart, M. y Carey. J (1995). "Incentives to Cultivate a Personal Vote: A Rank Ordering of Electoral Formulas.” Electoral Studies 14(4), pp. 417-439. 
Shugart, M. y Haggard, S. (2001). "Institutions and Public Policy in Presidential Systems", en Haggard. S, y McCubins M. (Eds.). Presidents, Parliaments, and Policy, 64-104. Nueva York: Cambridge University Press.

Stokes, W. (1945). "Parliamentary Government in Latin America”, American Political Science Review 39(3), pp. 522-536.

Uprimny, R. (2004). "The Constitutional Court and Control of Presidential Extraordinary Powers in Colombia”, en S. Gloppen, R. Gargarella y Skaar, E. (Eds.), Democratization and the Judiciary: The Accountability Function of Courts in New Democracies. Londres: Frank Cass, pp. 46-69.

Weldon, J. (1997). "Political Sources of Presidencialismo in Mexico", en S. Mainwaring y M. Shugart (Eds.), Presidentialism and Democracy in Latin America, pp. 2252-58. Nueva York: Cambridge University Press.

Zovatto, Daniel, y Jesus Orozco Henriquez (Eds.) (2008). Reforma Política y Electoral en América Latina, 1978-2007. Ciudad de México: UNAM. 